\title{
Nível de dependência de idosos em instituições de longa permanência
}

\author{
Level of dependency of elderly in institutions of long-term stay
}

Nivel de dependencia de ancianos en instituciones de larga permanencia

Jéssica Rodrigues Flores ${ }^{1 *}$, Herton Goerch ${ }^{1}$, Elenir Terezinha Rizzeti Anversa ${ }^{1}$, Cleci Lourdes Schmidt Piovesan-Rosanelli'.

\section{RESUMO}

Objetivo: Identificar o nível de dependência de idosos em duas instituições de longa permanência para idosos no município de Santa Maria/RS. Métodos: Trata-se de um trabalho de caráter quantitativo, descritivo e exploratório. A amostra foi elaborada com a participação de 44 idosos, de ambos os sexos, residentes em duas Instituições de Longa Permanência do município de Santa Maria. Foi realizado o Teste de Katz para medir a capacidade dos idosos de praticar as suas atividades diárias. Resultados: A análise descritiva apontou a média de idade de 78,9 anos, com predominância do sexo feminino, $84 \%$, da religião católica $84,09 \%$, com ensino fundamental incompleto, $50 \%$ e, no uso de medicamento, predominância de uso de 5 até 10 medicamentos, com a parcela de $45 \%$ dos institucionalizados. Concernente ao grau de dependência, $84 \%$ apresentam-se dependência para banhar-se e vestir-se; $56,82 \%$ para a mobilidade; $75 \%$ para o uso do sanitário; $68 \%$ para controle da continência. Conclusão: A maioria dos idosos das instituições analisadas foi considerada dependente para o desenvolvimento das atividades de vida diária.

Palavras-chave: Enfermagem, Envelhecimento, Saúde do idoso.

\begin{abstract}
Objective: To identify the level of dependency of the elderly in two long-term institutions for the elderly in the city of Santa Maria / RS. Methods: This is a work of a quantitative, descriptive and exploratory character. The sample was elaborated with the participation of 44 elderly people, of both sexes, residing in two Long-Term Institutions in the municipality of Santa Maria. The Katz test was performed to measure the ability of the elderly to practice their daily activities. Results: The descriptive analysis showed an average age of 78.9 years, with a predominance of females, $84 \%$, of the Catholic religion $84.09 \%$, with incomplete elementary education, $50 \%$ and, in the use of medication, predominance of use from 5 to 10 drugs, with $45 \%$ of those institutionalized. Concerning the degree of dependence, $84 \%$ are dependent for bathing and dressing; $56.82 \%$ for mobility; $75 \%$ for the use of the toilet; $68 \%$ for continence control. Conclusion: Most of the elderly in the analyzed institutions were considered dependent for the development of activities of daily living.
\end{abstract}

Keywords: Nursing, Aging, Elderly care.

\section{RESUMEN}

Objetivo: Identificar el nivel de dependencia de las personas mayores en dos instituciones de larga duración para personas mayores en la ciudad de Santa María / RS. Métodos: Se trata de un trabajo de carácter cuantitativo, descriptivo y exploratorio. La muestra se elaboró con la participación de 44 ancianos, de ambos sexos, residentes en dos Instituciones de Larga Duración en el municipio de Santa María. La prueba de Katz se realizó para medir la capacidad de los ancianos para practicar sus actividades diarias. Resultados: El

${ }^{1}$ Faculdade Integrada de Santa Maria (FISMA), Santa Maria - RS. *E-mail: jessicarflores@outlook.com 
análisis descriptivo arrojó una edad promedio de 78,9 años, con predominio del sexo femenino, $84 \%$, de religión católica $84,09 \%$, con educación primaria incompleta, $50 \%$ y, en el uso de medicamentos, predominio del uso de 5 a 10 fármacos, con el $45 \%$ de los institucionalizados. En cuanto al grado de dependencia, el $84 \%$ son dependientes para bañarse y vestirse; $56,82 \%$ para movilidad; $75 \%$ para el uso del baño; $68 \%$ para el control de la continencia. Conclusión: La mayoría de los adultos mayores de las instituciones analizadas fueron considerados dependientes para el desarrollo de las actividades de la vida diaria.

Palabras clave: Enfermería, Envejecimiento, Salud del anciano.

\section{INTRODUÇÃO}

De acordo com a Organização Mundial da Saúde (OMS), é considerado idoso o indivíduo com 60 anos ou mais e o habitante de país desenvolvido com ou acima de 65 anos. O envelhecimento é uma realidade mundial que implica diversas modificações sociais, estruturais e culturais. A população de idosos é a que mais eleva no Brasil, e existem mais de 28 milhões de pessoas nessa faixa etária, número que representa $13 \%$ da população do país (IBGE, 2018).

O envelhecimento saudável não é somente ausência da doença, mas o bem-estar em relação a si mesmo e à sociedade em que se está inserido. Ter uma boa alimentação, sono regular e atividade física são características fundamentais, mas, além disso, o idoso precisa de relacionamentos na vida cotidiana, seja com amigos ou familiares que o acolham, o respeitem e façam com que se sintam úteis, estimulando 0 autocuidado e a autoestima (BRÊTAS AC, 2014).

Além disso, na Constituição Brasileira, no Estatuto do Idoso, Lei n 10.741 (2003), artigo 3o, está definido que $\mathrm{o}$ apoio às pessoas idosas é de responsabilidade da família, sociedade e estado, os quais devem assegurar a sua participação na comunidade, defender sua dignidade e bem-estar, assim como garantir seu direito à vida (BRASIL, 2017).

O aumento das doenças crônicas e o processo fisiológico do envelhecimento levam a uma diminuição da capacidade funcional, o que pode gerar um comprometimento na independência dos idosos, tornando-os mais frágeis e carentes de ajuda para sobreviver e cumprir as suas atividades da vida diária. A dependência é considerada o estado em que o idoso se encontra por razões ligadas à falta ou perda da autonomia. Devido à dificuldade na realização das tarefas cotidianas e no estado mental, os idosos são encaminhados a instituições de longa permanência (MARIANO PP, et al., 2015).

Sendo assim, para conhecer o grau de dependência de idosos, tem-se a necessidade de avaliar a sua capacidade funcional pelo teste de Katz, que é utilizado por profissionais da área de enfermagem para avaliar o grau de dependência dos mesmos. Por meio dessa avaliação, podemos entender o grau de dependência e independência em realizar as atividades de vida diária, porém sabemos que existe uma escassez de programas sociais e da saúde voltada para os idosos a domicílio que tem uma dependência total, de modo que seus familiares necessitam de auxílio para realizar o manejo com este idoso. Por isso, em muitos casos, isso leva à escolha da institucionalização, ou seja, a internação precoce desses idosos em instituições de longa permanência (MARINHO LM, et al., 2015).

Motivos que podem ser considerados para o encaminhamento desses idosos às Instituição de Longa Permanência para Idosos (ILPI) é a diminuição de independência e do desempenho funcional do idoso, em que a análise dessas condições é um importante indicador da saúde (MARIANO PP, et al., 2015).

Considera-se importante avaliar qual o nível de dependência dos idosos internados em Instituições de Longa Permanência. Existem escalas que avaliam atividades básicas da vida diária como banho, vestir, banheiro, transferência, continência e alimentação. A avaliação do nível de dependência possibilita dimensionar a carga de trabalho enfrentados pelos cuidadores dos idosos e auxilia os profissionais de saúde no planejamento da assistência. Desse modo, o presente estudo estruturou-se com o objetivo de identificar o nível de dependência de idosos de duas Instituições de Longa Permanência no município de Santa Maria/RS. 


\section{MÉTODOS}

Desenvolveu-se uma pesquisa de campo de abordagem quantitativo e descritivo, do tipo transversal. A pesquisa foi realizada no município de Santa Maria, no estado do Rio Grande do Sul, no Brasil, que contava com 280.505 habitantes em 2018, segundo estimativas do IBGE, bem como é considerada uma cidade média e de grande influência na região central do estado.

O presente estudo foi desenvolvido em duas Instituições de longa permanência para idosos (ILPI), ambas de caráter particular, e foram classificadas como instituição $A$ e instituição $B$, são mistas e serão apresentadas de forma pormenorizada no relatório de pesquisa.

Participaram do estudo 44 idosos. Neste processo, foi utilizada uma amostra não probabilística, do tipo intencional pois é aquela em que a seleção dos elementos da população para compor a amostra depende ao menos em parte do julgamento do pesquisador ou do entrevistador no campo (MATTAR FN, 2001).

Os fatores de inclusão para a participação da pesquisa foram: idosos que habitam na ILPI, que foram convidados e aceitaram participar da pesquisa e assinaram o Termo de Consentimento livre e Esclarecido (TCLE). No caso dos pacientes que não tiveram condição cognitiva e/ou clínica para deliberar o aceite foi solicitada autorização de familiares ou responsáveis pelos mesmos.

Foi encaminhado ofício com apresentação dos objetivos para a Enfermeira responsável da ILPI, solicitando autorização dessa instituição para a realização da pesquisa de campo. A partir do aceite para realização da pesquisa junto aos idosos, o projeto foi registrado na Diretoria de Pós-graduação, pesquisa e Extensão da Faculdade Integrada de Santa Maria (FISMA). Na sequência, o referido projeto foi submetido na Plataforma Brasil, para ser designado ao Comitê de Ética em Pesquisa (CEP). Obteve o Parecer Consubstanciado de aprovação, 3.921.069.

Diante da liberação do CEP, houve contato com a instituição e os idosos e/ou responsáveis pelos mesmos, onde houve a exposição dos objetivos da pesquisa, bem como esclarecimento da importância do estudo. Na sequência, iniciou-se a pesquisa por meio do agendamento de data e horário de acordo com a disponibilidade dos idosos e da instituição. A coleta de dados ocorreu entre janeiro e abril de 2020. Foram utilizados um questionário sociodemográfico e clínico dos idosos e o Teste de Katz (LINO VTS, et al., 2008).

Inicialmente, foi aplicado o questionário para identificar o perfil sociodemográfico e clínico, por meio da entrevista com os idosos e/ou consulta aos prontuários dos mesmos para coletar dados referentes a: idade, sexo, escolaridade, ramo de atividade, doença de base e uso de dispositivos, medicação contínua. $\mathrm{Na}$ sequência, aplicado o Teste de Katz, para avaliar as condições do idoso para realizar as atividades de vida diária e, assim, avaliou-se seu grau de dependência e independência classificadas parcial ou total para efetivação de cuidados básicos.

O Teste de Kartz é um instrumento usado para verificar as habilidades que os idosos têm para realizar as atividades diárias, de modo a verificar o grau de dependência ou de independência. O referido instrumento, que varia de zero a seis pontos, quanto mais baixo escore maior a dependência. Tais atividades são as rotineiras, como o banho, colocar a própria roupa, ir ao banheiro, se alimentar, entre outras, de modo que para cada atividade pode haver um nível de dependência diferente (LINO VTS, et al., 2008).

A avaliação dá-se de uma nota de zero a seis, em que zero indica a independência total para realizar determinada atividade, aumentando o número ao mesmo tempo em que o grau da dependência vai aumentando, sendo seis a dependência total (LINO VTS, et al., 2008). Após a coleta das informações por meio dos instrumentos aplicados, os dados foram digitados e organizados em planilhas do Excel.

Para o tratamento dos dados foram utilizados métodos de Estatística Descritiva para organizar, resumir e descrever os aspectos importantes de um conjunto de características observadas ou comparar tais características entre os dados coletados. A descrição dos dados também tem como objetivo identificar anomalias, até mesmo resultante do registro incorreto de valores, e dados dispersos, aqueles que não seguem a tendência geral do restante do conjunto. 
No decorrer da pesquisa, foi observado rigorosamente o que consta na Resolução n466/2012 do Conselho Nacional de Saúde do Ministério da Saúde (BRASIL, 2012). Foram tomados os devidos cuidados na formulação do roteiro da entrevista, de forma a preservar os participantes de maneira que não permitiu a sua identificação. A todos os participantes foram esclarecidos os objetivos e métodos e todos os elementos da pesquisa.

\section{RESULTADOS}

A amostra de estudo se constituiu com 44 indivíduos distribuídos em duas instituições denominadas de "instituição A" e "instituição B", sendo que 30 (68,18\%) residiam na instituição A e 14 (31,82\%) na instituição $B$, todos da raça branca (100\%). Destaca-se que dois dos idosos convidados não aceitaram participar da pesquisa.

Em relação à faixa etária, a idade dos idosos das duas instituições variou entre 60 e 100 anos, 23\% destes possuíam mais de 90 anos sendo que apenas um idoso encontrava-se na faixa etária de 100 anos. A média foi de 78,9 anos.

$\mathrm{Na}$ instituição A, entre as faixas etárias de 60 e 70 anos, constatou-se $30 \%$ dos participantes; entre 70 e 80 anos o percentual foi de 10\%; entre 80 e 90 anos, foi de $33 \%$, sendo a predominante nesta instituição e, por fim, entre as faixas etárias de 90 e 100 anos, foi registrado o percentual de $27 \%$.

No que se refere à instituição $B$, entre as faixas etárias de 60 e 70 anos e 70 e 80 , registrou-se $21,5 \%$ dos avaliados; entre 80 e 90 anos, foi de $43 \%$, também sendo a faixa etária predominante e, por fim, entre 90 e 100 anos, foi registrado o percentual de $14 \%$.

Em relação ao sexo, observou-se que há predominância do sexo feminino, tanto na instituição $A$ quanto na Instituição B. Dos 44 participantes, 32 são do sexo feminino, o equivalente a $72,73 \%$ dos entrevistados, sendo apenas $27,27 \%$ do sexo masculino, ou seja, 12 participantes.

Em relação à escolaridade, a análise revelou que $20,45 \%$ dos voluntários das duas instituições avaliadas possuem ensino superior completo; $2,28 \%$ com ensino superior incompleto; $11,37 \% \mathrm{com}$ o ensino fundamental completo. A metade dos institucionalizados, ou seja, $50 \%$ possuem o ensino fundamental incompleto e $15,90 \%$ são não alfabetizados.

Quanto à renda, verificou-se que 4,54\% dos idosos recebem de três a quatro salários mínimos (nacional); $29,51 \%$ recebem de um a dois salários mínimos; $36,36 \%$ recebem até um salário mínimo e $29,51 \%$ dos idosos não recebem salário mínimo.

Concernente à situação trabalhista dos idosos, percebeu-se que, antes de serem institucionalizados, temse que $100 \%$ dos que eram do ramo de atividade do lar, donas de casa, ou não trabalhavam são do sexo feminino. Neste contexto, os que trabalhavam como comerciários e caminhoneiros são na totalidade do sexo masculino, parcela de $100 \%$. No ramo de atividade rural, dos $42,85 \%$ que trabalhavam neste segmento são do sexo feminino e $57,15 \%$ são do sexo masculino, ainda, na profissão de professor(a), chegou-se à porcentagem de $75 \%$ do sexo feminino e $25 \%$ do sexo masculino. Por fim, salienta-se que nas outras profissões houve variação do sexo dos participantes.

Relativo às comorbidades de base, os dados obtidos caracterizam a doença de Alzheimer com a de maior incidência entre os idosos, tendo um percentual de 38,63\%. Desse percentual, $82,35 \%$ são do sexo feminino e $17,65 \%$ são do sexo masculino, o que determina a predominância da doença nas idosas institucionalizadas. Ademais, $11,36 \%$ dos voluntários apresentam hipertensão, com prevalência de $80 \%$ nas mulheres e $20 \%$ nos homens, e $11,36 \%$ tiveram AVC, com a soma de $60 \%$ do sexo feminino e $40 \%$ masculino.

Nas categorias de idade, distúrbio mental e algum tipo de transtorno psiquiátrico, apresentam a porcentagem de $6,82 \%$ cada, sendo que nos quesitos idade e transtorno psiquiátrico, todos os que apresentam essas doenças são do sexo feminino, já com distúrbio mental $66,7 \%$ são do sexo feminino e $33,3 \%$ do sexo masculino. 
A doença de Parkinson apresenta 4,55\% de doenças base, sendo 50\% de cada sexo. Por fim, tem-se que na categoria outras, que engloba doenças menos incidentes, apresenta o percentual de $13,64 \%$, dentro desta taxa $66,7 \%$ dos idosos são do sexo masculino e $33,3 \%$ do sexo feminino.

Concernente ao uso de medicação contínua pelos idosos de ambos os sexos e das duas instituições do estudo, a maior prevalência sobre estes foi o uso de até 10 remédios controlados, efetivando $45 \%(20), 43 \%$ (10) fazem uso de até 5 medicamentos, $7 \%$ (3) fazem uso de apenas um medicamento e $5 \%$ (2) fazem uso de mais de 10 medicamentos.

É sabido que o desgaste físico, e o declínio são inevitáveis e desencadeiam desafios adaptativos para o idoso, em particular quando institucionalizado. Entre os idosos pesquisados, coloca-se que $65,9 \%$ não fazem uso de dispositivo como sonda vesical de sistema fechado, traqueostomia, sonda gastrostomia, embora $41 \%$ deles sejam dependentes em todas as atividades básicas da vida, de acordo com o teste de Katz.

Observou-se que 16\% (7) dos idosos participantes são independentes e 84\% (37) são dependentes em pelo menos uma atividade cotidiana. Neste sentido, as variáveis apresentam que $11 \%$ (5) são dependentes em duas e cinco atividades, $21 \%$ (9) são dependentes em quatro atividades e $41 \%$ (18) dependentes em todas as atividades. E, de acordo com a classificação, houve uma predominância de dependência em todas as atividades. Sendo que na população do sexo feminino essa parcela é de $88,89 \%$ (16) e no sexo masculino esse índice é de $11,11 \%$ (2).

$\mathrm{Na}$ avaliação do grau de dependência, constatou-se que 84,1\% (37) dos idosos residentes das duas instituições eram dependentes para banhar-se e vestir-se, sendo estas as dependências mais acentuadas. $O$ uso de sanitário alcançou o índice de dependência de $72,72 \%$, ou seja, 32 dos idosos institucionalizados. No que tange à continência, incidiu o percentual de $61,4 \%$ de dependência, 27 dos sujeitos participantes.

Referente à Mobilidade, o percentual de dependência foi de $56,82 \%, 25$ dos indivíduos participantes da pesquisa e, por fim, no quesito Alimentação, houve incidência de dependência de $50 \%$ (22) dos voluntários avaliados. Nos itens Vestuário e Banho a dependência foi identificada predominantemente no sexo feminino, atingindo $84,4 \%$ (27) das idosas institucionalizadas.

Já no item Uso de sanitário, verificou-se que $75 \%$ das idosas eram dependentes para realizar a atividade e $66,7 \%$ dos idosos também se enquadram nessa condição, sendo a proporcionalidade de 24 mulheres e 8 homens. No quesito Mobilidade, observou-se que também houve predominância no sexo feminino $(59,4 \%)$, ou seja, 19 idosas, enquanto do sexo masculino a metade possui essa dependência (50\%).

Com relação à Continência, verificou-se que 68,75\% (22 das 32 idosas residentes) não tinham o completo controle das eliminações urinária e intestinal; e 41,7\%, 05 idosos, também padeciam da mesma problemática. No que tange às dependências no item Alimentação, constatou-se que a maioria da parcela que não conseguia se alimentar sem assistência era do sexo feminino, com $56,25 \%$ (18) dependentes, e quanto ao sexo masculino, $33,4 \%$ (4) necessitavam de auxílio total ou parcial para a realização da atividade.

\section{DISCUSSÃO}

Os resultados apresentados no presente estudo demonstram um predomínio e a maior sobrevida do sexo feminino entre os idosos institucionalizados, estando em conformidade com o padrão demográfico brasileiro (CHAIMOWICZ FA, 1997). Dados estes, semelhantes aos verificados em pesquisa que verificou o perfil sociodemográfico e funcional de idosos de quatro Instituições de Longa Permanência (FERREIRA LL, et al., 2012). Nesta senda, também se pode observar a similaridade com a análise de Ferreira DC e Yoshitome AY (2010), onde as variáveis apontaram que $84 \%$ dos residentes da ILPI eram do sexo feminino.

A predominância do gênero feminino é presumivelmente justificada pela contemporânea feminização da velhice, decursiva da maior expectativa de vida das mulheres no Brasil (AIRES M, et al., 2009). Ademais, pode-se pensar também na questão de que as mulheres se preocupam mais com a saúde do que os homens, de modo que estes apenas buscam os serviços de saúde quando já é tarde, o que pode acarretar a mortalidade precoce destes, tendo, portanto, mais mulheres idosas (SILVA PSR, et al., 2020). 
A institucionalização aparenta ser em grande proporção uma questão feminina, observada também em outras pesquisas, que evidenciam ter ocorrido um aumento acentuado nas taxas de institucionalização de idosas (ARAÚJO MOPH e CEOLIM MF, 2007; SOUZA SMST e SANTOS VLCG, 2007; DUCA GFD, et al., 2009). Pode-se relacionar, também, a predominância do gênero feminino ao fato de que as mulheres estão menos expostas a riscos de acidentes de trabalho, domésticos ou de trânsito, bem como são menos suscetíveis a homicídio ou suicídio (AMARAL SVA, et al., 2020).

A média de idade dos residentes institucionalizados foi congênere à identificada em outras pesquisas, sendo a média de 78,9 anos, caracterizando um perfil de idosos longevos, o que pode estar associado à estrutura etária da sociedade brasileira, a qual se deve ao crescimento da expectativa de vida, que é acompanhada pelo aumento das enfermidades crônicas, tornando mais constantes os agravamentos destas e também as internações (STORTI LB, 2013). Ademais, salienta-se que a maioria desses idosos possui o nível socioeconômico considerado baixo.

A baixa escolaridade prevaleceu, primordialmente, devido às dificuldades de acesso ao ambiente escolar do passado (LISBOA CR e CHIANCA TCM, 2012), correlacionadas às atividades agrícolas e de donas de casa, que foi predominante na amostra. Nesta senda, concernente à situação trabalhista dos idosos antes de serem institucionalizados, a totalidade de donas de casa e que não trabalhavam são do sexo feminino, o que enfatiza as questões culturais de desigualdade devido ao sexo.

Cumpre referenciar que o analfabetismo em idosos também representa uma realidade dos países que estão em desenvolvimento, podendo-se enquadrar aqui o Brasil, principalmente, porque os idosos atuais vivenciaram na infância um estágio social em que o ensino não era tido como uma prioridade (BORGES AM, et al., 2014).

Diante das variáveis analisadas, notou-se quanto ao uso de medicamentos pelos idosos participantes da pesquisa, a maioria faz uso de 5 até 10 medicamentos, ou seja, $45 \%$ dos voluntários, tendo neste certame o prevalecimento da parcela do sexo feminino.

Ainda, pode-se destacar que em relação às patologias associadas ao envelhecimento funcional, grande parte dos idosos apresentaram mais de uma, o que corrobora com este estudo. Na pesquisa de Torres GV e Reis LA (2020), as análises trazem que a prevalência das doenças crônicas que vem ocasionando o aumento das incapacidades física e/ou mental entre os idosos institucionalizados, além de ser um risco para a qualidade de vida desses sujeitos.

Ainda, é possível se perceber que estes idosos demandam, progressivamente, cuidados de enfermagem mais qualificados, justificados pelo nível de dependência. Os idosos que estão institucionalizados despertam uma preocupação ainda maior pela fragilidade social, psicológica e física em que podem se encontrar (LISBOA CR e CHIANCA TCM, 2012).

São vários os motivos associados ao comprometimento das habilidades operacionais dos idosos, como a idade avançada, o gênero feminino, várias enfermidades, declínio cognitivo, assim como a pouca incidência de contato social (SANTOS GS e CUNHA ICKO, 2013). Nesta pesquisa, houve a constatação que ser dona de casa e com o grau de escolaridade baixo foram associados a maior grau de dependência. Esses dados podem estar correlacionados ao ínfimo convívio social dos idosos.

As variáveis denotaram que os institucionalizados mais dependentes para as atividades de vida diária eram os que possuíam mais de uma doença de base. Essas diretrizes podem estar associadas ao processo de envelhecimento, que, grande parte das vezes, não é caracterizado como um período saudável e que prevaleça a independência. A incidência de comorbidades crônicas não transmissíveis é relativamente maior entre os idosos, sendo apurada como uma das causas primordiais de incapacidade e dependência destes (PINTO MF, et al., 2009).

Cumpre destacar que é de suma importância que o ambiente da instituição de longa permanência seja apropriado para o idoso, devido à atenuação da capacidade adaptativa face às demandas ambientais, concomitantemente, é de extrema importância disponibilizar estímulos e desafios de forma que isto proporcione o desenvolvimento de formas de compensação. Uma vez que ao manter o controle total de fatores 
de queda, as instituições diminuem as chances de mobilidade por parte dos idosos, tendo como resultante o declive da capacidade de mobilidade e em uma dependência maior para a execução das atividades de vida diárias (COZZANI M e CASTRO EM, 2005).

Por fim, tem-se que os resultados da pesquisa apontam para a necessidade de motivação dos idosos no ambiente da instituição de longa permanência, deixando de lado os planos individualizados para incitar o potencial de autocuidado, a fim de que sejam independentes o máximo possível.

\section{CONCLUSÃO}

A maioria dos idosos das instituições analisadas foi considerada dependente para o desenvolvimento das atividades de vida diária. A parcela maior de institucionalizados ocorreu entre o sexo feminino, assim como 0 maior grau de dependência e idade avançada. Os resultados mostram a necessidade de planejamento por parte da equipe para que planeje atividades para aumentar a capacidade funcional, estimulando 0 autocuidado e independência, respeitando sempre o tempo e o limite de cada idoso. Os resultados também mostram que a interferência das instituições no autocuidado e atividades rotineiras acabam por vezes, prejudicando e agravando o grau de dependência dos pacientes, já que essas atividades, quando limitadas, acabam diminuindo a competência de autocuidado dos pacientes.

\section{REFERÊNCIAS}

1. AMARAL SVA, et al. Conhecimento e comportamento de um grupo de idosos frente às infecções sexualmente transmissíveis. Revista Eletrônica Acervo Saúde, 2020; 12(9)e3891.

2. AIRES M, et al. O Grau de dependência e características de pessoas idosas institucionalizadas. Rev. Bras. Ciênc. Envelhec. Hum., 2006; 3:79-91.

3. ARAÚJO MOPH, CEOLIM MF. Avaliação do grau de independência de idosos residentes em instituições de longa permanência. Rev Esc Enferm USP., 2007; 41(3): 378-85.

4. BORGES AM, et al. Autopercepção de saúde em idosos residentes em um município do interior do Rio Grande do Sul. Rev. Bras. Geriatr. Gerontol., 2014; 17(1): 79-86.

5. BRASIL. CARDENETA DE SAÚDE DA PESSOA IDOSA. Brasília, 2017, BRASIL. Manual do Ministério de Saúde 2020.

6. BRASIL. Conselho Nacional de Saúde. Resolução oㅜ 466, de 12 de dezembro de 2012. Brasília, Ministério da Saúde, 2012. Disponível em: https://conselho.saude.gov.br/resolucoes/2012/Reso466.pdf.

7. BREETAS AC. Cuidadores de idosos e o sistema único de saúde. v.3, Brasileira (DF): Revista Brasileira, 2014.

8. CHAIMOWICZ F. A saúde dos idosos brasileiros às vésperas do século XXI: problemas, projeções e alternativas. Rev. Saúde Pública. 1997; 31(2): 184-200.

9. COZZANI M, CASTRO EM. Estratégias adaptativas durante o andar na presença de obstáculos em idosos: impacto da institucionalização e da condição física. Rev Bras Educ Fis Esp., 2005; 19(1): 49-60.

10. DUCA GFD, et al. Incapacidade funcional para atividades básicas e instrumentais da vida diária em idosos. Rev. Saúde Pública. 2009; 43(5): 796-805.

11. FERREIRA LL, et al. Perfil sócio-demográfico e funcional de idosos institucionalizados. Estud. Interdiscipl. Envelhec. 2012; 17(2): 373-86.

12. FERREIRA DC, YOSHITOME AY. Prevalence and features of falls of institutionalized elders. Rev. Bras. Enferm. 2010;63(6):991-7.

13. LINO VTS, et.al. Adaptação transcultural da Escala de Independência em Atividades da Vida Diária (Escala de Katz). Cad. Saúde Pública, 2008; 24(1).

14. LISBOA CR, CHIANCA TCM. Perfil epidemiológico, clínico e de independência funcional de uma população idosa institucionalizada. Rev. Bras Enferm. 2012; 65(3): 482-7.

15. MARIANO PP, et al. Organização do trabalho de enfermagem nas instituições de longa permanência para idosos: relação com o prazer e sofrimento laboral. Florianópolis: Texto Contexto Enferm, 2015.

16. MATTAR FN. Pesquisa de marketing.3 ed. São Paulo: Atlas, 2001.

17. MARINHO LM, et al. Grau de dependência de idosos residentes em instituição de longa permanência. Rev. Gaúcha. Enferm, 2013; 34.

18. PELEGRIN AKAP, et al. Idosos de uma instituição de longa permanência de Ribeirão Preto: níveis de capacidade funcional. Arquivos de Ciências da Saúde, 2008; 15(4): 182-188.

19. PINTO MF, et al. Qualidade de vida de cuidadores de idosos com doença de Alzheimer. Acta. Paul. Enferm. 2009; 22(5): 652-7.

20. SANTOS GS, CUNHA ICKO. Avaliação da capacidade funcional de idosos para o desempenho das atividades instrumentais da vida diária: um estudo na atenção básica em saúde. Ver. Enferm. Cent. o Min. 2013; 3(3): 820-8.

21. SILVA PSR, et al. Perfil clínico-epidemiológico de pacientes portadores de hanseníase em um município do Maranhão. Revista Eletrônica Acervo Saúde, 2020; 12(8): e3468.

22. SOUZA DMST, SANTOS VLCG. Fatores de risco para o desenvolvimento de úlceras por pressão em idosos institucionalizados. Rev. Latinoam. Enferm. 2007; 15(5): 958-64.

23. STORTI LB, et al. Fragilidade de idosos internados na clínica médica da unidade de emergência de um hospital geral terciário. Texto Contexto Enferm. 2013; 22(2): 452-9.

24. TORRES GV, REIS LA. Assessment of functional capacity in elderly residents of outlying area in the hinterland of Bahia/ Northeast Brazil. Arquivos de Neuro-Psiquiatria. São Paulo, 2020; 68(1): 39-43. 